Published in final edited form as:

Transl J Am Coll Sports Med. 2020 ; 5(12): . doi:10.1249/tjx.0000000000000138.

\title{
Associations Between Habitual Sedentary Behavior and Endothelial Cell Health
}

\author{
Andrea T. Duran ${ }^{1,2}$, Carol Ewing Garber ${ }^{1}$, Ipek Ensari², Daichi Shimbo ${ }^{2}$, Keith M. Diaz ${ }^{2}$ \\ ${ }^{1}$ Department of Biobehavioral Sciences, Teachers College, Columbia University, 525 W. $120^{\text {th }}$ \\ Street, New York, NY, 10027, United States; \\ ${ }^{2}$ Center for Behavioral Cardiovascular Health, Columbia University Irving Medical Center, 622 W. \\ $168^{\text {th }}$ Street, New York, NY, 10032, United States.
}

\section{Abstract}

Endothelial dysfunction is a mechanism that may explain the link between prolonged sedentary time and cardiovascular disease. However, the relation between habitual sedentary behavior (SED) and endothelial function has yet to be explored.

Purpose--The purpose of this study was to examine the association of accelerometer-measured SED with markers of endothelial cell health.

Methods-—Healthy adult participants ( $\mathrm{n}=83 ; 43.4 \%$ male; $25.5 \pm 5.8$ years old) were examined. SED was measured for 7-days by accelerometer. Endothelial function measures included endothelium-dependent vasodilation (EDV); endothelial microparticles (EMPs) [CD62E+ and CD31+/CD42- EMPs]; and endothelial progenitor cells (EPCs) [CD34+/CD133+/KDR+ and CD34+/KDR+EPCs]. Participants were classified as having low or high SED based on a median split.

Results-—Participants in the low and high SED group spent a mean \pm SD of $8.6 \pm 1.1$ and $11.1 \pm$ $1.0 \mathrm{~h} /$ day in SED, respectively. No significant differences between the low and high SED groups were detected in mean [95\% confidence interval (CI)] EDV (2.51 [2.21-2.81] vs. 2.36 [2.07-2.64], $p=0.50$ ), EMPs (CD62E+: 6.70 [6.55-6.84] vs. 6.56 [6.42-6.69], $p=0.20$; CD31+/CD42-: 6.26 [6.10-6.42] vs. 6.18 [6.03-6.33], $p=0.50)$, or EPCs (CD34+/KDR+: $11.91[9.23-14.48] \times 10^{-2}$ vs. $\left.14.87[12.41-17.32] \times 10^{-2}, p=0.13\right) ; \mathrm{CD} 34+/ \mathrm{CD} 133+/ \mathrm{KDR}+: 1.84[1.28-2.39] \times 10^{-2}$ vs. 2.17 $\left.[1.64-2.70] \times 10^{-2}, p=0.43\right)$.

Conclusions--Among healthy adults, habitual SED was not associated with markers of endothelial cell health.

Correspondence: Andrea T. Duran, Columbia University Irving Medical Center, 622 West $168^{\text {th }}$ Street, PH 9-313, New York, NY 10032, Phone: 212-678-3325, Fax: 212-678-3322, atd2127@cumc.columbia.edu.

Disclosure of Interest: The authors report no conflict of interest. The results of the present study do not constitute endorsement by the American College of Sports Medicine.

Trial registration number: NCT01909895 (clinicaltrials.gov) 


\section{Keywords}

sedentary behavior; endothelial function; endothelial microparticles; endothelial progenitor cells; cardiovascular health

\section{Introduction}

Prolonged sedentary time is associated with incident cardiovascular disease (CVD) and mortality, independent of moderate-vigorous physical activity (MVPA) $(1,2)$. However, the mechanisms underlying these associations have not been elucidated. Endothelial dysfunction, an early pathogenic process underlying atherosclerosis, is a possible putative mechanism $(3,4)$. The sitting posture (the primary sedentary posture) promotes muscle inactivity of the lower extremities and changes in the angles at which the femoral and popliteal arteries run, causing bends within the arterial tree (5). These physiological conditions elicit hemodynamic changes that include blood pooling in the legs, decreased thigh and calf blood flow, and augmented turbulent blood flow in the deformed arterial segments $(5,6)$. For these reasons, it is thought that prolonged sitting may promote atherosclerosis and increased CVD risk by exposing the endothelium to a pro-atherogenic milieu, facilitating dysfunction of the endothelium over time $(7,8)$.

Supportive of this hypothesis, experimental evidence has shown that prolonged sitting blights endothelial-dependent vasodilation (EDV) in the popliteal and femoral arteries in 1 to 6 hours $(9,10)$. This sitting induced endothelial dysfunction, however, has shown to be restored with even light muscular activity (e.g., leg fidgeting), which brings into question the long-lasting effects of prolonged sitting on the vasculature outside the context of an acute laboratory setting $(10,11)$. Moreover, the laboratory based models employed in existing studies are limited because 1) acute periods of sitting in the lab over a single day (or in most cases a few hours) is not indicative of chronic exposure to sitting, and 2) the control condition (uninterrupted sitting for hours at a time) does not have real world generalizability (12). Thus, it is unclear if chronic exposure to such conditions with prolonged sitting contributes to endothelial dysfunction.

Studies have defined endothelial dysfunction solely as an impairment in EDV. This narrow focus provides insight concerning only one aspect of endothelial function. Lab-based investigations have elucidated cellular measures of endothelial dysfunction, including endothelial cell (EC) injury and diminished EC reparative capacity. Cellular measures include circulating endothelial microparticles (EMPs), a measure of EC injury, and circulating endothelial progenitor cells (EPCs), a measure of EC reparative capacity (13). The purpose of the current study was to comprehensively examine whether habitual sedentary time is associated with markers of endothelial function, including EDV, circulating levels of EMPs, and circulating levels of EPCs, in a cohort of healthy adults. We hypothesized that participants with greater sedentary time would exhibit worse EC health (i.e., lower EDV, higher circulating EMPs, lower circulating EPCs). 


\section{Materials and Methods}

\subsection{Study Participants:}

Healthy adult participants were enrolled into the Putative Mechanisms Underlying

Myocardial Infarction Onset and Emotions (PUME) study, a laboratory-based, single-blind, randomized controlled experimental study conducted from August 2013 to May 2017

$(\mathrm{N}=280)$. As described elsewhere in detail, PUME was designed to examine the impact of negative emotion (i.e., anger, anxiety and sadness) induction tasks on EC health (14). Inclusion criteria included adults $\geq 18$ years of age. Exclusion criteria included individuals with any: (a) chronic medical condition including prevalent CVD and traditional CVD risk factors including history of hypertension, diabetes, dyslipidemia; (b) active smoking; (c) medication use including over-the-counter drugs and herbal medications; or (d) self-reported history of psychosis, mood disorders, or personality disorder diagnoses.

Instrumented measures of sedentary behavior were collected in a subsample of PUME participants from September 2014 to June 2017. All active PUME participants were invited to complete a 7-day accelerometer protocol: 163 were invited of whom 94 consented to participate and were able to be scheduled. Excluding those with missing data $(n=3)$ or with non-adherent accelerometer wear time ( $<3$ days with $\geq 10$ hours of wear; $n=8)$, useable data were available from 83 participants. Characteristics of PUME participants included and those excluded in the present analyses are presented in the supplemental content (see Table 1, Supplemental Digital Content 1, which demonstrates characteristics of participants included and excluded from the current study). The PUME study protocol was approved by the institutional review board at Columbia University Medical Center. All participants provided written informed consent.

\subsection{Procedures:}

Participants came into the laboratory on two occasions. The first visit entailed collection/ measurement of endothelial markers, for which the participants were instructed to arrive at 08:30 am following a fast from the previous midnight, and to refrain from any strenuous exercise in the 12 hours prior to their visit. Upon arrival, they were escorted to a temperature-controlled room and seated in a comfortable chair. A 20-gauge intravenous catheter was inserted into an antecubital vein of the dominant arm. Afterwards, the participant was instrumented with the EndoPAT2000 ${ }^{\mathrm{TM}}$ device and instructed to relax for 30 min. Following this rest, EDV assessment was completed. Blood was then drawn into collection tubes. One citrated tube was used to measure circulating EMPs, and one EDTA tube was used to measure EPCs. EDV and blood draws were collected at 5 different time points; the current study only analyzed the baseline measures (time point 1) collected prior to the negative emotion induction task.

A second visit was scheduled 7-14 days after the initial laboratory visit. At this visit, participants were fitted with the activPAL (V.3, PAL Technologies, Glasgow, UK), a thighworn triaxial accelerometer and inclinometer that has been validated for determining physical activity intensities, posture (sitting/lying, standing or stepping), and sedentary time in healthy adults (15-18). Participants were instructed to wear the device continuously for 7 
days and to not remove the monitor unless it was to be fully submerged in water (e.g., swimming, bath). Participants were also asked to complete a sleep and wear-time log sheet to record daily sleep ('lights out') and wake times, and times when the device was removed (if any).

\subsection{Accelerometer Processing:}

Time-stamped 15-second epoch data files were exported using the activPAL software for subsequent processing and analysis in SAS 9.4. Non-wear and sleep time recorded in the logs were excluded from analyses. For each participant, minutes of sedentary time, standing time, light-intensity physical activity (LIPA, defined as 1.5-2.99 metabolic equivalents [METs] derived from stepping cadence), and MVPA (defined as $\geq 3$ METs derived from stepping cadence) were summed for each day and averaged across the number of valid days ( $\geq 10 \mathrm{~h}$ of wear) to derive 'per day' values (15). Sedentary and MVPA bouts were also quantified. A sedentary bout was defined as consecutive epochs in which the activPAL registered no standing or stepping events of any length. An MVPA bout was defined as any stepping period of $\geq 10$ minutes for which each consecutive epoch had a stepping cadence assigned an activity intensity of $\geq 3$ METs. We corrected for the influence of variation in wear time by standardizing sedentary time using the residuals obtained when regressing sedentary time on wear time $(19,20)$. As a result, sedentary time is expressed as the mean predicted sedentary time given a wear time of $16 \mathrm{~h} /$ day.

\subsection{Endothelial Cell Health Measures:}

EDV was determined using the reactive hyperemia index (RHI, unitless), which is measured as the transient increase in blood flow following a brief period of arterial occlusion. RHI was assessed using the EndoPAT $2000^{\mathrm{TM}}$, a validated peripheral arterial tonometry (PAT) device (21-23). EC injury and repair were assessed by measuring circulating EMPs and EPCs, respectively. Blood samples were prepared and processed using flow cytometry (BD FACS Calibur) and analyzed using previously published protocols (24-26). Two phenotypes of EMP were selected for analysis; those expressing CD62E+, which are phenotypic for EC activation, and those expressing CD31+, which are indicative of EC apoptosis (27). Similarly, two phenotypes of EPC were selected for analysis; those expressing CD34+/ $\mathrm{CD} 133+/ \mathrm{KDR}+$, and those expressing CD34+/KDR+. Data were gated on the mononuclear lymphocytic population, and 500,000 events are collected in the gated region for each sample. Data were expressed as percentages of the mononuclear lymphocytic populations that consist of CD34+/CD133+/KDR+ cells, and separately, CD34+/KDR+ cells. Details on EDV, EMP, and EPC measurements are available in the supplemental content (see Methods, Supplemental Digital Content 1, which provides detailed information on EDV, EMP, and EPC measurements).

Statistical Analyses: Participants were classified into high and low total sedentary time groups by a median split of $589 \mathrm{~min} /$ day of sedentary time. Descriptive statistics were computed to characterize the high and low sedentary time groups. For each EC variable, outliers were winsorized and thereafter transformed when appropriate. EMP data were natural log transformed. EPC data were square root transformed because zero was a possible value. 
Multivariable regression models were used to compare the levels of each EC variable (EDV, EMPs and EPCs) between high and low sedentary time groups. Unadjusted models were first conducted. Subsequent models adjusted for age, sex, race, ethnicity and education (Model 1), and further adjusted for MVPA (Model 2) and body mass index (BMI) (Model 3 ). As a sensitivity analysis, all analyses were repeated with sedentary time expressed as a continuous variable in hours/day.

As some evidence suggests that prolonged, uninterrupted sedentary bouts (e.g. sitting for hours at a time) may potentially be the most hazardous form of sedentary behavior $(19,28$, 29), the above analyses were repeated examining mean sedentary bout duration (a measure of overall prolonged, uninterrupted sedentary behavior that has been linked to mortality) as the exposure variable (28). Participants were classified into high and low sedentary bout groups by a median split of $17.2 \mathrm{~min} / \mathrm{sedentary}$ bout. All analyses were conducted using SAS, version 9.4 (SAS Institute).

\section{Results}

\subsection{Participant Characteristics:}

In the overall sample ( $n=83$ ), sedentary behavior accounted for $61.7 \pm 10.2 \%$ of wear time, equivalent to $9.9 \pm 1.7$ hours/day over a 16-hour waking day. The mean $( \pm \mathrm{SD})$ mean sedentary bout duration was $18.7 \pm 7.4 \mathrm{~min} /$ bout. LIPA and MVPA accounted for $31.6 \pm 9.3$ $\%$ and $6.7 \pm 2.9 \%$ of wear time, respectively, equivalent to $306.6 \pm 95.8 \mathrm{~min} /$ day, and $64.5 \pm$ $28.0 \mathrm{~min} /$ day. Table 1 presents the characteristics of participants classified into the high and low sedentary time groups according to total sedentary time. Participants in the high sedentary group were more likely to be male and engaged in lower levels of standing, LIPA and MVPA. Characteristics of participants classified into high and low sedentary bout groups according to mean sedentary bout duration are shown in the supplemental content (see Table 2, Supplemental Digital Content 1, which demonstrates characteristics of participants classified into high and low sedentary bout groups according to mean sedentary bout duration).

\subsection{Sedentary Behavior and Endothelial Cell Health:}

Differences in the markers of EC health between the high and low total sedentary time groups are shown in Table 2. In unadjusted and adjusted models, there were no significant differences between high and low total sedentary time groups in EDV as indicated by the RHI. There were also no significant differences in circulating levels of EMPs (CD62E+ and CD31+/CD42-) and EPCs (CD34+/KDR+ and CD34+/CD133+/KDR+) in unadjusted and adjusted models. Similarly, when high and low sedentary groups were defined according to accumulation of sedentary time in prolonged, uninterrupted sedentary bouts (e.g. mean sedentary bout duration), there were no significant differences between the high and low groups for any of the endothelial measures (Table 3). In sensitivity analyses expressing total sedentary time and mean sedentary bout duration as continuous variables, there was no significant associations observed for any of the EC variables (see Table $3 \& 4$, Supplemental Digital Content 1 , which demonstrates results from the sensitivity analyses). 


\section{Discussion}

In this study of healthy adults, we evaluated several measures of EC health to examine the relation between ecological, habitual sedentary time and endothelial dysfunction. We hypothesized that participants with greater sedentary time would exhibit poorer EC health (i.e., lower EDV, higher circulating EMPs, lower circulating EPCs). Contrary to our hypothesis, we found that there were no statistically significant differences in measures of EDV, EC injury, or EC reparative capacity in participants with high compared with low volumes of accelerometer-measured sedentary time among a sample of healthy adults. These findings suggest habitual sedentary behavior may not incur CVD risk, in part, through endothelial dysfunction.

The contention that sedentary behavior induces CVD risk partially through vascular dysfunction is premised on experimental evidence, which has demonstrated that acute prolonged bouts of sitting elicits impairments in EDV, particularly of the legs (9). For example, Thosar and colleagues (2015) found that 3 hours of uninterrupted sitting impaired EDV and decreased mean and antegrade shear rates in the superficial femoral artery (10). Similarly, Restaino et al. (2015) found that 6 hours of prolonged, uninterrupted sitting impaired both microvascular dilator function (i.e., blood flow and velocity) and macrovascular dilator function (i.e., FMD) of the popliteal artery (5). Despite promising laboratory-based findings; the lack of real-world applicability (e.g., in some studies participants were carried from a chair to an exam table for EDV testing) and evidence that even small amounts of fidgeting can offset sitting-induced EDV are limitations of this current evidence and necessitate a need for testing of the sedentary behavior-endothelial function link under ecological conditions $(10,11)$. The present study addresses this evidence gap and provides some of the first data evaluating the associations between habitual levels of sedentary behavior and a panel of endothelial biomarkers.

Contrary to existing experimental evidence, our findings do not support a link between ecological sedentary behavior and endothelial dysfunction. Reasons for the discrepancy are unclear, but could be attributed to a number of factors. First, the mean sedentary bout duration was only $\sim 19$ min per bout in the present observational study; far less than the 1 to 6 hour sedentary bout durations used in laboratory-based studies. Second, we evaluated EDV in the upper extremities due to its strong correlation with EDV of the coronary arteries and demonstrated prognostic utility (30-32); characteristics not established with EDV in the commonly measured lower extremities. Thus, differences between the present observational study and previous laboratory-based studies could also be a result of upper extremity vs. lower extremity differences; albeit our EDV results are supported by our cellular measures of EC injury (EMPs) and repair (EPCs). Finally, differences may be attributed to study design (cross-sectional vs. acute induction of inactivity, wherein it is difficult to ascertain whether the observed effects are the result of increases in sedentary behavior or reductions in MVPA), inclusion of women (only men were studied in the previous experimental studies), and differences in the processing and analyzing of EMPs and EPCs (which widely vary from investigator to investigator). 
There are several strengths to our study. First, the current study utilized both EDV and cellular measures of endothelial function. Measuring EMPs and EPCs, in addition to EDV, enabled us to complete a comprehensive evaluation of EC health, which is essential to unveil the complex processes that underlie endothelial dysfunction (e.g., EC injury, repair and regeneration). Second, the activPAL was used for measuring habitual sedentary behavior. This device is widely considered the gold-standard measure of sedentary behavior because it is extremely accurate ( $296 \%$ ) and is one of the only devices capable of distinguishing motionless standing from sedentary time, thus allowing us to adhere to the consensus sedentary behavior definition, which includes both intensity of activity ( 4.5 METS) and position (sitting or reclining) (33). Finally, apparently healthy adults are an ideal population to study the effects of sitting on endothelial function as this population is generally free of overt chronic disease which could confound associations (e.g. those with multi-morbidities have poor physical function and are thus more sedentary) (13).

Limitations must be acknowledged when interpreting our study findings. First, this was a cross-sectional study, which limits our ability to evaluate the effect of sedentary time on endothelial function, as causation cannot be implied. Second, this is a small, single-center study, which may limit the generalizability of our findings and statistical power to detect significant differences between high and low sedentary groups. Thus, caution is warranted when interpreting our study findings given the possibility of a type II error.

In conclusion, this study demonstrated that there were no statistically significant differences in a comprehensive battery of endothelial function measures, including measures of EDV, EC injury, and EC reparative capacity, when comparing healthy adults that accumulated higher and lower levels of habitual sedentary behavior (both the total volume and accumulation in prolonged, uninterrupted bouts). These findings suggest that sedentary behavior accrued in ecological settings may not detrimentally influence systemic endothelial function in healthy adults.

\section{Supplementary Material}

Refer to Web version on PubMed Central for supplementary material.

\section{Acknowledgements:}

This work was supported by the National Heart, Lung, and Blood Institute (NHLBI) at the National Institutes of Health (NIH) under grant R01-HL116470, K24-HL125704, R01-HL116470-02S1 and R01-HL134985.

\section{References}

1. Wilmot EG, Edwardson CL, Achana FA, Davies MJ, Gorely T, Gray LJ, et al. Sedentary time in adults and the association with diabetes, cardiovascular disease and death: systematic review and meta-analysis. Diabetologia. 2012;55(11):2895-905. doi: 10.1007/s00125-012-2677-z. [PubMed: 22890825]

2. Biswas A, Oh PI, Faulkner GE, Bajaj RR, Silver MA, Mitchell MS, et al. Sedentary time and its association with risk for disease incidence, mortality, and hospitalization in adults: a systematic review and meta-analysis. Ann Intern Med. 2015;162(2):123-32. doi: 10.7326/M14-1651. [PubMed: 25599350] 
3. Versari D, Daghini E, Virdis A, Ghiadoni L, Taddei S. Endothelial dysfunction as a target for prevention of cardiovascular disease. Diabetes Care. 2009;32 Suppl 2:S314-21. 10.2337/dc09-S330. [PubMed: 19875572]

4. Ross R. Atherosclerosis--an inflammatory disease. N Engl J Med. 1999;340(2):115-26. 10.1056/ NEJM199901143400207. [PubMed: 9887164]

5. Restaino RM, Holwerda SW, Credeur DP, Fadel PJ, Padilla J. Impact of prolonged sitting on lower and upper limb micro- and macrovascular dilator function. Exp Physiol. 2015;100(7):829-38. 10.1113/EP085238. [PubMed: 25929229]

6. Padilla J, Sheldon RD, Sitar DM, Newcomer SC. Impact of acute exposure to increased hydrostatic pressure and reduced shear rate on conduit artery endothelial function: a limb-specific response. Am J Physiol Heart Circ Physiol. 2009;297(3):H1103-8. 10.1152/ajpheart.00167.2009. [PubMed: 19633210]

7. Hamilton MT, Hamilton DG, Zderic TW. Role of low energy expenditure and sitting in obesity, metabolic syndrome, type 2 diabetes, and cardiovascular disease. Diabetes. 2007;56(11):2655-67. 10.2337/db07-0882. [PubMed: 17827399]

8. Thosar SS, Johnson BD, Johnston JD, Wallace JP. Sitting and endothelial dysfunction: the role of shear stress. Med Sci Monit. 2012;18(12):RA173-80. Epub 2012/12/01. [PubMed: 23197245]

9. Padilla J, Fadel PJ. Prolonged sitting leg vasculopathy: contributing factors and clinical implications. Am J Physiol Heart Circ Physiol. 2017;313(4):H722-h8. 10.1152/ajpheart.00326.2017. [PubMed: 28733451]

10. Thosar SS, Bielko SL, Mather KJ, Johnston JD, Wallace JP. Effect of prolonged sitting and breaks in sitting time on endothelial function. Med Sci Sports Exerc. 2015;47(4):843-9. 10.1249/ mss.0000000000000479. [PubMed: 25137367]

11. Morishima T, Restaino RM, Walsh LK, Kanaley JA, Fadel PJ, Padilla J. Prolonged sitting-induced leg endothelial dysfunction is prevented by fidgeting. Am J Physiol Heart Circ Physiol. 2016;311(1):H177-82. 10.1152/ajpheart.00297.2016. [PubMed: 27233765]

12. Diaz KM, Howard VJ, Hutto B, Colabianchi N, Vena JE, Blair SN, et al. Patterns of Sedentary Behavior in US Middle-Age and Older Adults: The REGARDS Study. Med Sci Sports Exerc. 2016;48(3):430-8. doi: 10.1249/MSS.0000000000000792. [PubMed: 26460633]

13. Deanfield JE, Halcox JP, Rabelink TJ. Endothelial function and dysfunction: testing and clinical relevance. Circulation. 2007;115(10):1285-95. doi: 10.1161/CIRCULATIONAHA.106.652859. [PubMed: 17353456]

14. Ensari I, Burg MM, Diaz KM, Fu J, Duran AT, Suls JM, et al. Putative mechanisms Underlying Myocardial infarction onset and Emotions (PUME): a randomised controlled study protocol. BMJ Open. 2018;8(5):e020525. 10.1136/bmjopen-2017-020525.

15. Lyden K, Keadle SK, Staudenmayer J, Freedson PS. The activPALTM Accurately Classifies Activity Intensity Categories in Healthy Adults. Med Sci Sports Exerc. 2017;49(5):1022-8. 10.1249/mss.0000000000001177. [PubMed: 28410327]

16. Kozey-Keadle S, Libertine A, Lyden K, Staudenmayer J, Freedson PS. Validation of wearable monitors for assessing sedentary behavior. Med Sci Sports Exerc. 2011;43(8):1561-7. 10.1249/ MSS.0b013e31820ce174. [PubMed: 21233777]

17. Lyden K, Kozey Keadle SL, Staudenmayer JW, Freedson PS. Validity of two wearable monitors to estimate breaks from sedentary time. Med Sci Sports Exerc. 2012;44(11):2243-52. 10.1249/ MSS.0b013e318260c477. [PubMed: 22648343]

18. Grant PM, Ryan CG, Tigbe WW, Granat MH. The validation of a novel activity monitor in the measurement of posture and motion during everyday activities. Br J Sports Med. 2006;40(12):992-7. 10.1136/bjsm.2006.030262. [PubMed: 16980531]

19. Healy GN, Matthews CE, Dunstan DW, Winkler EA, Owen N. Sedentary time and cardiometabolic biomarkers in US adults: NHANES 2003-06. Eur Heart J. 2011;32(5):590-7. 10.1093/ eurheartj/ehq451. [PubMed: 21224291]

20. Willett W, Stampfer MJ. Total energy intake: implications for epidemiologic analyses. American journal of epidemiology. 1986;124(1):17-27. [PubMed: 3521261]

21. Bonetti PO, Pumper GM, Higano ST, Holmes DR, Kuvin JT, Lerman A. Noninvasive identification of patients with early coronary atherosclerosis by assessment of digital reactive hyperemia. Journal 
of the American College of Cardiology. 2004;44(11):2137-41. doi: 10.1016/j.jacc.2004.08.062. [PubMed: 15582310]

22. Goor DA, Sheffy J, Schnall RP, Arditti A, Caspi A, Bragdon EE, et al. Peripheral arterial tonometry: a diagnostic method for detection of myocardial ischemia induced during mental stress tests: a pilot study. Clin Cardiol. 2004;27(3):137-41. Epub 2004/03/31. [PubMed: 15049379]

23. Hansen AS, Butt JH, Holm-Yildiz S, Karlsson W, Kruuse C. Validation of Repeated Endothelial Function Measurements Using EndoPAT in Stroke. Front Neurol. 2017;8:178. 10.3389/ fneur.2017.00178. [PubMed: 28515707]

24. Garcia S, Chirinos J, Jimenez J, Muñoz FDC, Canoniero M, Jy W, et al. Phenotypic assessment of endothelial microparticles in patients with heart failure and after heart transplantation: switch from cell activation to apoptosis. The Journal of heart and lung transplantation. 2005;24(12):2184-9. [PubMed: 16364869]

25. Shimbo D, Rosenberg LB, Chaplin W, Zhao S, Goldensohn ER, Cholankeril M, et al. Endothelial cell activation, reduced endothelial cell reparative capacity, and impaired endothelial-dependent vasodilation after anger provocation. International journal of cardiology. 2013;167(3):1064. [PubMed: 23157814]

26. Urbich C, Dimmeler S. Endothelial progenitor cells characterization and role in vascular biology. Circulation research. 2004;95(4):343-53. [PubMed: 15321944]

27. Jimenez JJ, Jy W, Mauro LM, Horstman LL, Soderland C, Ahn YS. Endothelial microparticles released in thrombotic thrombocytopenic purpura express von Willebrand factor and markers of endothelial activation. British journal of haematology. 2003;123(5):896-902. [PubMed: 14632781]

28. Diaz KM, Howard VJ, Hutto B, Colabianchi N, Vena JE, Safford MM, et al. Patterns of Sedentary Behavior and Mortality in U.S. Middle-Aged and Older Adults: A National Cohort Study. Ann Intern Med. 2017;167(7):465-75. doi: 10.7326/M17-0212. [PubMed: 28892811]

29. Diaz KM, Goldsmith J, Greenlee H, Strizich G, Qi Q, Mossavar-Rahmani Y, et al. Prolonged, Uninterrupted Sedentary Behavior and Glycemic Biomarkers Among US Hispanic/Latino Adults: The HCHS/SOL (Hispanic Community Health Study/Study of Latinos). Circulation. 2017;136(15):1362-73. doi: 10.1161/CIRCULATIONAHA.116.026858. [PubMed: 28835368]

30. Matsuzawa Y, Kwon TG, Lennon RJ, Lerman LO, Lerman A. Prognostic Value of Flow-Mediated Vasodilation in Brachial Artery and Fingertip Artery for Cardiovascular Events: A Systematic Review and Meta-Analysis. J Am Heart Assoc. 2015;4(11). 10.1161/JAHA.115.002270.

31. Poredos P, Jezovnik MK. Testing endothelial function and its clinical relevance. J Atheroscler Thromb. 2013;20(1):1-8. [PubMed: 22972428]

32. Takase B, Uehata A, Akima T, Nagai T, Nishioka T, Hamabe A, et al. Endothelium-dependent flow-mediated vasodilation in coronary and brachial arteries in suspected coronary artery disease. Am J Cardiol. 1998;82(12):1535-9, A7-8. [PubMed: 9874063]

33. Gibbs BB, Hergenroeder AL, Katzmarzyk PT, Lee IM, Jakicic JM. Definition, measurement, and health risks associated with sedentary behavior. Med Sci Sports Exerc. 2015;47(6):1295-300. 10.1249/MSS.0000000000000517. [PubMed: 25222816] 
Table 1.

Characteristics of participants in the low and high total sedentary time groups $(\mathrm{n}=83)$.

\begin{tabular}{|c|c|c|c|}
\hline Variable & $\begin{array}{c}\text { Low } \\
(n=41)\end{array}$ & $\begin{array}{c}\text { High } \\
(n=42)\end{array}$ & P-Value \\
\hline \multicolumn{4}{|l|}{$\underline{\text { Participant Characteristics }}$} \\
\hline Age (years) & $26.7(7.3)$ & $24.4(3.4)$ & 0.08 \\
\hline $\operatorname{Men}(\%)$ & 31.7 & 54.7 & 0.03 \\
\hline Black Race $(\%)$ & 7.3 & 2.4 & 0.29 \\
\hline Hispanic Ethnicity (\%) & 29.3 & 21.4 & 0.40 \\
\hline Education & & & 0.16 \\
\hline$\leq$ High School Graduate $(\%)$ & 9.8 & 2.4 & \\
\hline Some College (\%) & 17.1 & 19.1 & \\
\hline College Graduate (\%) & 29.3 & 50.0 & \\
\hline Graduate/Professional School (\%) & 43.9 & 28.6 & \\
\hline Body Mass Index $\left(\mathrm{kg} / \mathrm{m}^{2}\right)$ & $24.7(4.1)$ & $23.5(3.8)$ & 0.16 \\
\hline \multicolumn{4}{|l|}{$\underline{\text { Accelerometer Characteristics }}$} \\
\hline Total Sedentary Time (mins/day) & $515.5(68.6)$ & $668.4(57.2)$ & $<0.001$ \\
\hline Mean Sedentary Bout Duration (mins/bout) & $15.1(3.7)$ & $22.3(8.3)$ & $<0.001$ \\
\hline Standing Time (mins/day) & $312.8(78.5)$ & $206.1(55.6)$ & $<0.001$ \\
\hline LIPA (mins/day) & $370.1(82.7)$ & $244.6(60.8)$ & $<0.001$ \\
\hline MVPA (mins/day) & $75.7(27.3)$ & $53.5(24.3)$ & $<0.001$ \\
\hline MVPA Bouts (mins/day) & $13.8(12.4)$ & $8.4(9.8)$ & 0.03 \\
\hline Wear Time (mins/day) & $961.8(63.0)$ & $971.9(56.0)$ & 0.44 \\
\hline Valid Wear Days & & & 0.15 \\
\hline $3-5$ days $(\%)$ & 5.0 & 0.0 & \\
\hline $6-7$ days $(\%)$ & 95.0 & 100.00 & \\
\hline
\end{tabular}

Data are presented as mean (standard deviation) or frequency

LIPA= light intensity physical activity; MVPA= moderate-vigorous physical activity.

MVPA Bouts= total minutes of MVPA accrued in bouts $\geq 10 \mathrm{~min}$; defined as any period of $\geq 10$ minutes for which each consecutive 15 -sec epoch had an activity intensity was 23 METs.

Median split cut-point for high and low total sedentary time groups was $589 \mathrm{~min} / \mathrm{day}$ 
Table 2.

Endothelial-dependent vasodilation, endothelial microparticles, and endothelial progenitor cells by total sedentary time groups $(n=83)$.

\begin{tabular}{|c|c|c|c|}
\hline Endothelial Cell Variable & $\begin{array}{c}\text { Low } \\
(n=41)\end{array}$ & $\underset{(n=42)}{\text { High }}$ & P-value \\
\hline \multicolumn{4}{|l|}{ Endothelial-dependent vasodilation ${ }^{a}$} \\
\hline Unadjusted & $2.53(2.24-2.83)$ & $2.32(2.08-2.55)$ & 0.25 \\
\hline Model 1 & $2.48(2.22-2.75)$ & $2.36(2.10-2.62)$ & 0.53 \\
\hline Model 2 & $2.46(2.18-2.75)$ & $2.38(2.10-2.66)$ & 0.72 \\
\hline Model 3 & $2.51(2.21-2.81)$ & $2.36(2.07-2.64)$ & 0.50 \\
\hline \multicolumn{4}{|l|}{ Endothelial Microparticles ${ }^{b}$} \\
\hline \multicolumn{4}{|l|}{$\mathrm{CD} 62 \mathrm{E}+($ counts $/ \mu \mathrm{l})$} \\
\hline Unadjusted & $6.72(6.58-6.86)$ & $6.55(6.43-6.66)$ & 0.06 \\
\hline Model 1 & $6.73(6.60-6.85)$ & $6.54(6.42-6.67)$ & 0.06 \\
\hline Model 2 & $6.71(6.57-6.84)$ & $6.56(6.43-6.70)$ & 0.19 \\
\hline Model 3 & $6.70(6.55-6.84)$ & $6.56(6.42-6.69)$ & 0.20 \\
\hline \multicolumn{4}{|l|}{ 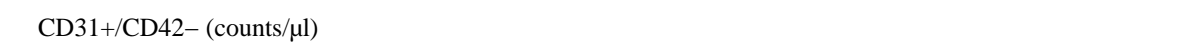 } \\
\hline Unadjusted & $6.23(6.06-6.40)$ & $6.24(6.11-6.36)$ & 0.96 \\
\hline Model 1 & $6.24(6.10-6.39)$ & $6.22(6.08-6.37)$ & 0.87 \\
\hline Model 2 & $6.28(6.12-6.43)$ & $6.19(6.04-6.34)$ & 0.46 \\
\hline Model 3 & $6.26(6.10-6.42)$ & $6.18(6.03-6.33)$ & 0.50 \\
\hline \multicolumn{4}{|l|}{ Endothelial Progenitor Cells ${ }^{c}$} \\
\hline \multicolumn{4}{|l|}{$\mathrm{CD} 34+/ \mathrm{KDR}+(\%)$} \\
\hline Unadjusted & $12.68(10.20-15.00) \times 10^{-2}$ & $14.18(11.86-16.50) \times 10^{-2}$ & 0.36 \\
\hline Model 1 & $13.11(10.77-15.44) \times 10^{-2}$ & $13.74(11.41-16.08) \times 10^{-2}$ & 0.71 \\
\hline Model 2 & $12.24(9.81-14.67) \times 10^{-2}$ & $14.62(12.19-17.05) \times 10^{-2}$ & 0.21 \\
\hline Model 3 & $11.91(9.23-14.48) \times 10^{-2}$ & $14.87(12.41-17.32) \times 10^{-2}$ & 0.13 \\
\hline \multicolumn{4}{|l|}{$\mathrm{CD} 34+/ \mathrm{CD} 133+/ \mathrm{KDR}+(\%)$} \\
\hline Unadjusted & $1.92(1.45-2.39) \times 10^{-2}$ & $2.13(1.66-2.61) \times 10^{-2}$ & 0.53 \\
\hline Model 1 & $1.90(1.40-2.39) \times 10^{-2}$ & $2.16(1.66-2.65) \times 10^{-2}$ & 0.47 \\
\hline Model 2 & $1.92(1.40-2.45) \times 10^{-2}$ & $2.13(1.60-2.66) \times 10^{-2}$ & 0.62 \\
\hline Model 3 & $1.84(1.28-2.39) \times 10^{-2}$ & $2.17(1.64-2.70) \times 10^{-2}$ & 0.43 \\
\hline
\end{tabular}

Data are presented as mean (95\% confidence interval) for unadjusted analyses or estimated marginal mean (95\% confidence interval) for adjusted analyses.

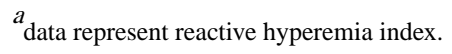

$b_{\text {data are natural } \log \text { transformed. }}$

$c_{\text {data are square root transformed. }}$

Model 1: Adjusted for age, sex, race, ethnicity and education

Model 2: Adjusted for covariates in model 1 plus moderate-vigorous physical activity.

Model 3: Adjusted for covariates in model 2 plus body mass index

Transl J Am Coll Sports Med. Author manuscript; available in PMC 2021 October 01. 
Median split cut-point was $589 \mathrm{~min} /$ day for total sedentary time 
Table 3.

Endothelial-dependent vasodilation, endothelial microparticles, and endothelial progenitor cells by median split of mean sedentary bout duration $(n=83)$.

\begin{tabular}{|c|c|c|c|}
\hline Endothelial Cell Variable & $\begin{array}{c}\text { Low } \\
(n=41)\end{array}$ & $\begin{array}{c}\text { High } \\
(n=42)\end{array}$ & P-value \\
\hline \multicolumn{4}{|c|}{ Endothelial-dependent vasodilation ${ }^{a}$} \\
\hline Unadjusted & $2.45(2.16-2.74)$ & $2.40(2.15-2.65)$ & 0.81 \\
\hline Model 1 & $2.37(2.11-2.63)$ & $2.48(2.22-2.73)$ & 0.58 \\
\hline Model 2 & $2.36(2.09-2.62)$ & $2.49(2.23-2.75)$ & 0.49 \\
\hline Model 3 & $2.39(2.12-2.67)$ & $2.46(2.20-2.73)$ & 0.74 \\
\hline \multicolumn{4}{|l|}{ Endothelial Microparticles $b$} \\
\hline \multicolumn{4}{|l|}{ 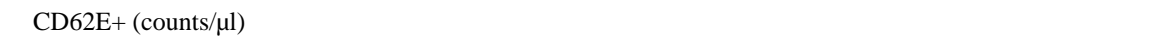 } \\
\hline Unadjusted & $6.70(6.55-6.85)$ & $6.57(6.45-6.68)$ & 0.15 \\
\hline Model 1 & $6.70(6.58-6.83)$ & $6.56(6.44-6.69)$ & 0.14 \\
\hline Model 2 & $6.69(6.56-6.82)$ & $6.57(6.44-6.70)$ & 0.22 \\
\hline Model 3 & $6.69(6.55-6.82)$ & $6.56(6.44-6.69)$ & 0.21 \\
\hline \multicolumn{4}{|l|}{ 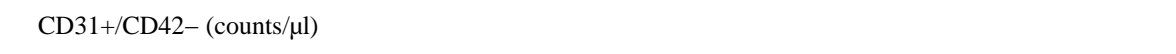 } \\
\hline Unadjusted & $6.25(6.09-6.40)$ & $6.22(6.08-6.37)$ & 0.81 \\
\hline Model 1 & $6.23(6.11-6.40)$ & $6.21(6.07-6.35)$ & 0.68 \\
\hline Model 2 & $6.27(6.12-6.41)$ & $6.20(6.06-6.34)$ & 0.54 \\
\hline Model 3 & $6.23(6.09-6.38)$ & $6.20(6.06-6.35)$ & 0.77 \\
\hline \multicolumn{4}{|l|}{ Endothelial Progenitor Cells ${ }^{c}$} \\
\hline \multicolumn{4}{|l|}{$\mathrm{CD} 34+/ \mathrm{KDR}+(\%)$} \\
\hline Unadjusted & $12.68(10.33-15.03) \times 10^{-2}$ & $14.14(11.85-16.43) \times 10^{-2}$ & 0.38 \\
\hline Model 1 & $13.29(10.94-15.63) \times 10^{-2}$ & $13.56(11.28-15.85) \times 10^{-2}$ & 0.87 \\
\hline Model 2 & $12.93(10.59-15.28) \times 10^{-2}$ & $13.90(11.61-16.18) \times 10^{-2}$ & 0.57 \\
\hline Model 3 & $12.83(10.38-15.28) \times 10^{-2}$ & $14.02(11.66-16.37) \times 10^{-2}$ & 0.51 \\
\hline \multicolumn{4}{|l|}{$\mathrm{CD} 34+/ \mathrm{CD} 133+/ \mathrm{KDR}+(\%)$} \\
\hline Unadjusted & $2.07(1.59-2.55) \times 10^{-2}$ & $1.98(1.51-2.45) \times 10^{-2}$ & 0.79 \\
\hline Model 1 & $2.08(1.58-2.57) \times 10^{-2}$ & $1.98(1.49-2.46) \times 10^{-2}$ & 0.78 \\
\hline Model 2 & $2.11(1.60-2.61) \times 10^{-2}$ & $1.95(1.46-2.44) \times 10^{-2}$ & 0.67 \\
\hline Model 3 & $2.15(1.62-2.67) \times 10^{-2}$ & $1.88(1.38-2.39) \times 10^{-2}$ & 0.49 \\
\hline
\end{tabular}

Data are presented as mean (95\% confidence interval) for unadjusted analyses or estimated marginal mean (95\% confidence interval) for adjusted analyses.

a data represent reactive hyperemia index.

$b_{\text {data are natural } \log \text { transformed. }}$

$c_{\text {data are square root transformed. }}$

Model 1: Adjusted for age, sex, race, ethnicity and education

Model 2: Adjusted for covariates in model 1 plus moderate-vigorous physical activity.

Model 3: Adjusted for covariates in model 2 plus body mass index

Transl J Am Coll Sports Med. Author manuscript; available in PMC 2021 October 01. 
Median split cut-point was $17.2 \mathrm{~min} /$ bout for mean sedentary bout duration. 